\title{
Influence of attractive van der Waals interactions on the optimal excitations in thermocapillary-driven spreading
}

\author{
Jeffrey M. Davis and Sandra M. Troian* \\ Department of Chemical Engineering, Princeton University, Princeton, New Jersey 08544-5263 \\ (Received 9 March 2002; revised manuscript received 12 November 2002; published 30 January 2003)
}

\begin{abstract}
Recent investigations of microfluidic flows have focused on manipulating the motion of very thin liquid films by modulating the surface tension through an applied streamwise temperature gradient. The extent to which the choice of contact line model affects the flow and stability of such thermocapillary-driven films is not completely understood. Regardless of the contact line model used, the linearized disturbance operator corresponding to the evolution of the film height is non-normal, and a generalized non-modal stability analysis is required. Surprisingly, early predictions of frontal instability that stemmed from conventional modal analysis of thermocapillary flow on a flat, infinite precursor film showed excellent agreement with experiment. Within the more rigorous framework provided by a generalized stability analysis, this work investigates the transient dynamics and amplification of optimal disturbances subject to a finite precursor film generated by attractive van der Waals forces. Convergence of the disturbance growth rates and perturbed shapes to the asymptotic solutions obtained by conventional linear stability analysis occurs early in the spreading process. In addition, the level of transient disturbance amplification is minimal. The equations governing thermocapillary-driven spreading exhibit a small degree of non-normality, which explains the source of agreement between modal theory and experiment. The more rigorous generalized stability analysis presented here, however, affords critical insight into the types of disturbances leading to maximum unstable growth and the exact influence of the contact line model used.
\end{abstract}

DOI: 10.1103/PhysRevE.67.016308

PACS number(s): 47.15.Fe, 47.20.Dr, 47.54.+r, 68.15.+e

\section{INTRODUCTION}

The recent focus on microscale flow phenomena and microfluidic devices $[1,2]$ has generated interest in controlling the motion of thin liquid films through modulation of surface forces. One such technique exploits the temperature dependence of the surface tension to regulate the magnitude and direction of flow $[3,4]$. Application of a temperature gradient to a solid substrate produces a gradient in the surface tension of the supported liquid film. This induced thermocapillary stress forces liquid to spread from warmer to cooler regions. For thin films spreading on a uniform and chemically homogeneous surface, a capillary ridge develops at the leading edge. In analogy to other driven spreading systems [5], this ridge undergoes capillary breakup to produce a parallel array of spreading rivulets that resemble drip marks in wet paint films.

Direct comparison of the features of unstable flow between theory and experiment or between different theoretical models has been hampered by two issues. The first issue centers on the choice of contact line model (and parameter range) used to relieve the stress singularity at the moving front caused by the no-slip boundary condition. Choices include the application of an infinite precursor film of constant thickness ahead of the contact line, the inclusion of attractive van der Waals forces in the governing equation for the film height, or various slip boundary conditions at the liquid-solid interface. In one of the few studies to compare directly the

\footnotetext{
*Electronic address: stroian@princeton.edu; URL: www.princeton.edu/ stroian
}

effect of different contact line models on the flow and stability of films driven to spread by gravity or centrifugation [6], it was shown that there was little difference in the shape of the base states, the dispersion curves, and the eigenfunctions generated by either the infinite flat precursor film $[7,8]$ or the Greenspan slip condition [9] for parameter ranges leading to comparable film thicknesses. The second issue involves the type of analysis used in characterizing the linear stability of the flow. While numerical predictions of the most unstable wavelength computed from conventional modal linear stability show excellent agreement with experiment for thermally driven flows $[10,11]$, it is now recognized [12] that since the linearized disturbance operator is nonnormal, a generalized nonmodal analysis is required. Recently, a nonmodal analysis for thermocapillary-driven spreading on a flat and infinite precursor film has shown that the disturbance growth rates and evolved shapes corresponding to the optimal perturbations rapidly asymptote to the predictions of modal theory with minimal transient growth [12]. Whether these results can be generalized to a system subject to a more realistic contact line model is of significant interest.

Implementation of a more realistic or self-consistent precursor film, as for example, one generated by attractive van der Waals forces, was first suggested by Huh and Scriven [13], who studied lubrication coating flows near the solidliquid-vapor line of contact. They noted that long-range forces become increasingly dominant upon approach to the moving contact line and derived the asymptotic shape of the film thickness profile in the van der Waals dominated regime. Golovin et al. [14] recently implemented a similar approach by including an attractive van der Waals term directly into the lubrication equations for thermocapillary spreading. While this study demonstrates the stabilizing influence of the 
van der Waals term, it relies on conventional modal analysis. These results are therefore formally valid only for the linearized equations in the infinite time limit [15]. Nonetheless, relieving the contact line singularity by regarding the van der Waals forces as an additional pressure gradient on the spreading film is more realistic than simply patching the liquid film to an infinite and featureless precursor layer. Furthermore, the magnitude of the Hamaker constant which controls the degree of disjoining pressure in the film can be obtained from experimental data, thereby eliminating the use of arbitrary values for the precursor film thickness.

This present work extends the earlier study of Golovin et al. [14] by investigating the generalized non-modal stability of thermocapillary-spreading films subject to attractive van der Waals forces. Because the validity of predictions of modal stability theory cannot be assured a priori for systems governed by non-normal disturbance operators, the analysis described below is the appropriate method for determining the stability of free surface, lubrication flows. To facilitate comparison with the modal results, a similar parameter range to that used by Golovin et al. is investigated throughout this work.

\section{GOVERNING EQUATIONS}

Consider the upwards thermocapillary spreading of a completely wetting, Newtonian liquid film of density $\rho$ and viscosity $\eta$ along a substrate inclined from the horizontal by an angle $\theta$. The liquid is supplied from a source at constant flux, and a constant thermal gradient is applied along the substrate such that the temperature decreases in the direction of spreading. The governing equation for the film height in the lubrication approximation has been derived elsewhere $[10,16]$, so only the final result is presented here. Near the front of the spreading film, in the inner region of the flow where capillary forces are comparable to viscous and thermocapillary forces, the evolution equation is given by

$$
\begin{aligned}
h_{t}+ & \left(\frac{\tau h^{2}}{2 \eta}\right)_{x}-\left[\frac{\rho g \sin (\theta) h^{3}}{3 \eta}\right]_{x}+\nabla \cdot\left[\frac{h^{3}}{3 \eta} \nabla\{-\rho g \cos (\theta) h\right. \\
& \left.\left.+\gamma_{0} \nabla^{2} h+A_{0} h^{-3}\right\}\right]=0
\end{aligned}
$$

where $\hat{x}$ indicates the streamwise direction of flow, $\hat{y}$ indicates the direction transverse to the flow, $g$ is the gravitational acceleration constant, and $A_{0}$ is the Hamaker constant. The second and third terms represent contributions to the liquid flux arising from the positive thermocapillary force and the negative force due to gravitational drainage. The terms within brackets signify contributions from hydrostatics, capillary forces due to interfacial curvature for $|\nabla \widetilde{h}|^{2}$ $\ll 1$, and attractive van der Waals interactions, respectively. In thermocapillary-driven systems, the viscosity $\eta$ of the liquid can vary spatially but variations in liquid density are typically much smaller and can be ignored. For the short migration distances studied experimentally $[17,18]$, the viscosity of the film is relatively constant, and this simplification is utilized in the analysis below. The temperature depen- dence of the surface tension $\gamma$, however, plays a critical role and is well approximated by the linear relation

$$
\gamma=\gamma_{0}+\sigma\left(T-T_{0}\right)
$$

where $\sigma=d \gamma / d T$, is constant (and negative) over a large temperature range [19]. Application of a constant thermal, gradient, therefore, produces a constant shear stress $\tau$ $=(d \gamma / d T)(d T / d x)$ at the air-liquid interface which drives the spreading film toward the coolest region of the substrate.

Equation (1) is converted to dimensionless form by introducing the variable transformations:

$$
\begin{gathered}
\chi=-\frac{x}{l}, \\
\zeta=\frac{y}{l}, \\
\bar{h}=\frac{h}{h_{c}}, \quad \text { and } \\
\bar{t}=\frac{t}{l / U_{c}} .
\end{gathered}
$$

The dynamic capillary length $l=h_{c} /(3 \mathrm{Ca})^{1 / 3}$ defines the spatial extent near the moving front over which the capillary forces due to interfacial curvature compete with the thermocapillary and viscous forces. The capillary number is defined by $\mathrm{Ca}=\eta U_{c} / \gamma_{o}$, where $U_{c} \equiv h_{c} \tau / \eta$ is the characteristic thermocapillary speed at the air-liquid interface [20]. The quantity $h_{c}$ denotes the characteristic film thickness far from the contact line, which can be determined from matching the film thickness to the outer region of the flow. If gravitational drainage is significant, as is the case with much thicker climbing films, then the characteristic flow speed includes the retarding term $-h_{c}^{2} \rho g \sin (\theta) /(3 \eta)$ [11]. For the range of parameters considered below, for which gravitational drainage is negligible, the value of $h_{c}$ is established by the film thickness emerging from the meniscus region at the liquid source [21-23].

The dimensionless form of Eq. (1) is

$$
h_{t}+(B h-1) h h_{\chi}+\nabla \cdot\left[h^{3} \nabla\left(-D h+\nabla^{2} h+V h^{-3}\right)\right]=0 \text {, }
$$

where $\nabla \equiv \hat{\chi} \partial_{\chi}+\hat{\zeta} \partial_{\zeta}$ and subscripts denote partial differentiation with respect to $\chi, \zeta$, or $t$. From hereon all the overbars are dropped for convenience. Three parameters appear in Eq. (4), namely

$$
\begin{gathered}
B=\frac{\rho g \sin (\theta) h_{c}}{\tau}, \\
D=\left(\frac{\rho g \cos (\theta) h_{c}^{2}}{\gamma_{0}}\right)\left(\frac{3 \tau h_{c}}{\gamma_{0}}\right)^{-2 / 3}, \quad \text { and } \\
V=\left(\frac{A_{0}}{\gamma_{0} h_{c}^{2}}\right)\left(\frac{3 \tau h_{c}}{\gamma_{0}}\right)^{-2 / 3} .
\end{gathered}
$$


The parameter $B$ characterizes the relative strength of gravitational drainage to the (upwards) thermocapillary force. For a horizontally spreading film, $B=0$. For the vertically climbing films examined by Cazabat et al. [17,22] $B=O\left(10^{-2}\right)$. The parameter $D$ describes the strength of the hydrostatic pressure relative to the thermocapillary force. Based on available experimental data $[17,18,22,24], D$ is estimated to be of $O\left(10^{-3}\right)$. In what follows, $B$ and $D$ will therefore be neglected, and only the influence of the parameter $V$, which characterizes the effect of van der Waals forces relative to thermocapillary forces, is studied. The parameter range for $V$ used in this study is similar to that first explored by Golovin et al. [14] and is representative of estimates derived from the experimental data of Ludviksson and Lightfoot [24] and Cazabat et al. [17,22].

\section{STEADY TRAVELING WAVE SOLUTIONS}

The spreading process can be viewed from a reference frame moving at constant dimensionless speed $c$ by introducing the change of variable $\xi=\chi+c t$. The steady solution to Eq. (4) is a traveling wave $h=h_{0}(\xi)$ subject to the boundary conditions $h_{0}(\xi \rightarrow \infty)=1, h_{0 \xi}(\xi \rightarrow \infty)=0, h_{0}(\xi \rightarrow-\infty)=b$, and $h_{0 \xi \xi \xi \xi}(\xi \rightarrow-\infty)=0$, where $b \geqslant 0$ is a constant representing the asymptotic thickness of the van der Waals film. These constraints yield a third-order equation for the evolution of the base state

$$
h_{0}^{3} h_{0 \xi \xi \xi}-3 V h_{0 \xi} h_{0}^{-1}-\frac{1}{2} h_{0}^{2}+c h_{0}-K=0
$$

where $c=(1+b) / 2$ and $K=-b / 2$. If $b>0$, then the film height $h_{0}$ approaches the asymptotic film thickness $b$ exponentially fast. This fast decay is the reason for which van der Waals forces are sometimes simply mimicked by matching the front of a spreading film to an infinite precursor layer of constant thickness $[10,11,12]$. For $b=0$, the precursor film is finite and to first order $h_{0} \sim-1 / \xi$ as $\xi \rightarrow-\infty$. The precursor model with $b=0$ forms the focus of this work.

In this limit, Eq. (6) becomes

$$
h_{0}^{3} h_{0 \xi \xi \xi}-3 V h_{0 \xi} h_{0}^{-1}-\frac{1}{2} h_{0}^{2}+\frac{1}{2} h_{0}=0 .
$$

A Taylor expansion about $h_{0}=0$ yields an implicit equation for the asymptotic behavior in the precursor region

$$
\xi+\frac{6 V}{h_{0}}+6 V \ln \left(\frac{1}{h_{0}}-1\right)=0,
$$

where the arbitrary constant of integration is set to zero because the governing equation is translationally invariant. The series expansion is somewhat involved because of the presence of logarithmic terms, but Eq. (8) can also be obtained by neglecting the term $h_{0}^{3} h_{0 \xi \xi \xi}$ in Eq. (7).

\section{A. Numerical solutions of the unperturbed height profile}

The base state solutions $h_{0}(\xi)$ to Eq. (7) are determined by using a standard shooting scheme [25]. The integration is

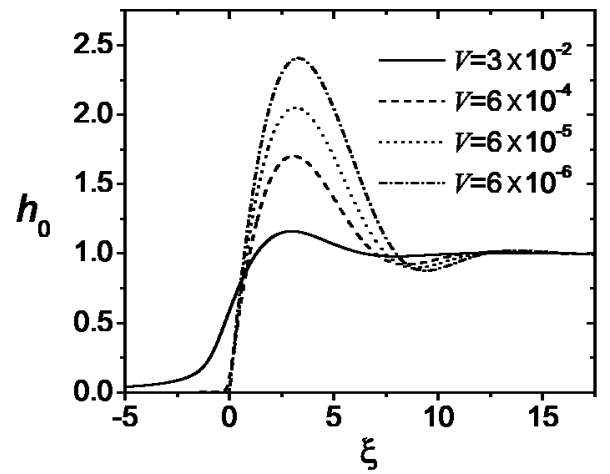

(a)

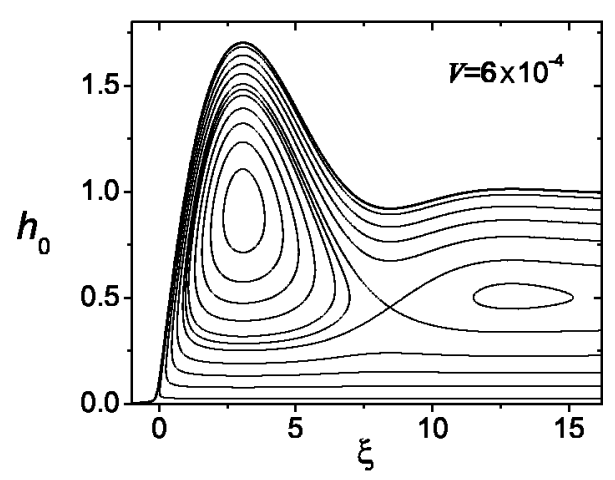

(b)

FIG. 1. (a) Numerical solution of the steady state film profile, $h_{0}(\xi)$, for parameter values $V=3 \times 10^{-2}, 6 \times 10^{-4}, 6 \times 10^{-5}$, and $6 \times 10^{-6}$. The influence of the van der Waals forces is apparent in the transition from the capillary ridge to the precursor film. (b) The streamline pattern in the moving reference frame $\xi=\chi+c t$ for the case $V=6 \times 10^{-4}$.

started with $h_{0} \approx 1$, and iteration of the solution continues until the precursor film profile matches onto the asymptotic shape described by Eq. (8). The choice $b=0$ enforces a van der Waals precursor film whose thickness vanishes in the limit $\xi \rightarrow-\infty$. Because the continuum model loses validity as the film thickness thins to molecular dimensions, the film is numerically truncated at the location at which the dimensional film height is approximately $O(1 \mathrm{~nm})$. Once the precursor film is sufficiently thin, the exact location at which the film is taken to end had a negligible effect on the stability results presented in Secs. III B 1 and IV.

Typical solutions for the film height in the inner region are shown in Fig. 1(a). For small values of the parameter $V$, the film thickness rapidly vanishes near the contact line. Smaller values of $V$ correspond to smaller values of the parameter $b$ used in models employing a flat and infinite precursor $[10,11,12]$. Numerical comparison of the base state profiles generated with these two contact line models indicates that a value $V=6 \times 10^{-4}$ corresponds to $b \approx 0.05$. As the magnitude of $V$ is increased, the liquid is increasingly attracted to the solid substrate with a subsequent decrease in the amplitude of the capillary ridge. For sufficiently large values of $V$, the capillary ridge can be altogether suppressed, resulting in a spreading film that is asymptotically stable. In this limit, which requires film thicknesses in the nanometer 
range, the flow speed due to van der Waals forces is comparable to the thermocapillary speed.

The streamfunction $\psi(\xi, z)$ in the moving reference frame can be determined from the equation

$$
\begin{aligned}
\psi(\xi, z)= & {\left[\frac{1}{3} z^{3}-h_{0}(\xi) z^{2}\right]\left[h_{0}(\xi)_{\xi \xi \xi}-3 V h_{0}(\xi)^{-4} h_{0}(\xi)_{\xi}\right] } \\
& -\frac{1}{3} z^{2}
\end{aligned}
$$

once the solution $h_{0}(\xi)$ is known. Here, $z$ denotes the dimensionless coordinate normal to the spreading plane. The cubic polynomial results from simplifications introduced through the lubrication approximation. Selected streamlines are plotted in Fig. 1(b). In the reference frame moving with the film, the flow recirculates in the region of the capillary ridge. Two streamlines intersect at the $\xi$ position, where the height profile undergoes a local minimum behind the capillary ridge. This intersection occurs at the $z$ value, where the local streamwise velocity vanishes. A very similar pattern of streamlines was obtained by Goodwin [26] when solving the full Stokes' equation for a falling film. In that case also, there is a crossing of streamlines at the stagnation point corresponding to the local minimum in film thickness behind the capillary ridge, although this streamline was not explicitly shown in that work. The close agreement between the streamline pattern derived from the Stokes' equation and that derived from lubrication theory further confirms the validity of the lubrication approximation for thin viscous coating flows.

\section{B. Linear stability of traveling wave solutions}

Profiles with large capillary ridges as shown in Fig. 1(a) can undergo sinusoidal fingering instabilities with well defined wave number $q$ in the transverse direction $(\hat{\zeta})$. The dimensionless wave number is based on the dynamic capillary length $l$ as defined earlier. Substitution of the perturbed wave forms $h(\xi, \zeta, t)=h_{0}(\xi)+\varepsilon h_{1}(\xi, \zeta, t)$ with $\varepsilon \ll 1$, where

$$
h_{1}(\xi, \zeta, t)=G(\xi, t) \exp (i q \zeta)
$$

into Eq. (4) yields the evolution equation for the streamwise disturbance function $G(\xi, t)$ :

$$
\begin{aligned}
\frac{\partial G}{\partial t}+ & \left(\frac{1}{2} h_{0 \xi}-\frac{24 V h_{0 \xi}^{2}}{h_{0}^{3}}+\frac{12 V h_{0 \xi \xi}}{h_{0}^{2}}+\frac{3 q^{2} V}{h_{0}}+q^{4} h_{0}^{3}\right) G \\
& +\left(\frac{15 V h_{0 \xi}}{h_{0}^{2}}+\frac{1}{2} h_{0}-2 U-3 q^{2} h_{0}^{2} h_{0 \xi}\right) G_{\xi}+\left(\frac{-3 V}{h_{0}}\right. \\
& \left.-2 q^{2} h_{0}^{3}\right) G_{\xi \xi}+\left(3 h_{0}^{2} h_{0 \xi}\right) G_{\xi \xi \xi}+h_{0}^{3} G_{\xi \xi \xi \xi}=0
\end{aligned}
$$

This equation is subject to the decay boundary conditions $G, G_{\xi} \rightarrow 0$ as $\xi \rightarrow \pm \infty$. This linear equation can be cast in operator form as

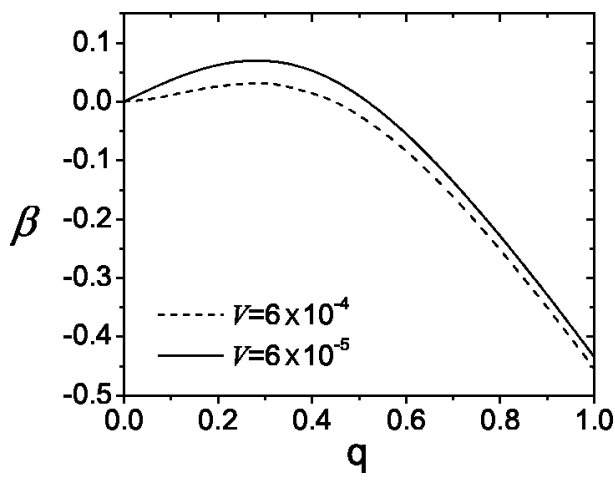

FIG. 2. Dispersion curves, $\beta(q)$, from eigenvalue analysis corresponding to the base state profiles shown in Fig. 1 for $V=6$ $\times 10^{-4}$ and $V=6 \times 10^{-5}$.

$$
\frac{\partial G}{\partial t}=\mathbf{A}(\xi) G
$$

where A denotes the autonomous linearized disturbance operator and $G(t)$ represents the state of the system at time $t$. Because of the spatial dependence of the base state solution $h_{0}(\xi)$ shown in Fig. 1(a), the operator $\mathbf{A}(\xi)$ is non-normal and therefore does not commute with its adjoint, i.e., $\mathbf{A A}^{\dagger}$ $\neq \mathbf{A}^{\dagger} \mathbf{A}$. While the stability of a normal system for all times $t$ is strictly governed by the eigenspectrum of $\mathbf{A}$, this is not necessarily the case for non-normal systems. It is now widely recognized that the modal spectrum for non-normal operators only determines the asymptotic stability (of the linearized equations) as $t \rightarrow \infty$. A more general analysis is required for examining the stability of the flow at finite times [15].

\section{Asymptotic solutions}

The asymptotic solution to Eq. (11) in the limit $t \rightarrow \infty$ can be further specified according to the exponential form $G(\xi, t)=H(\xi) \exp (\beta t)$, where $\beta$ denotes the (dimensionless) disturbance growth rate [15]. The numerical solutions for $H(\xi)$ and $\beta(q)$ are found by discretizing Eq. (11) using a central difference scheme and applying a standard QR algorithm in MATLAB 5.3 to determine the eigenvalues and eigenfunctions. The dispersion curves, corresponding to the base state height profiles for $V=6 \times 10^{-4}$ and $V=6 \times 10^{-5}$ shown in Fig. 1, are plotted in Fig. 2. The system is asymptotically unstable for disturbances whose wave numbers lie in the range $0<q \leqslant 0.5$. The most unstable mode occurs at a value $q_{\max } \approx 0.30$.

\section{Transient nonmodal solutions}

The general solution to Eq. (12) is found by similarly discretizing Eq. (11) according to a central difference scheme. The number of grid points used to determine the elements comprising matrix A ranged from 3000 to 5000 . The decay boundary conditions determine the entries in the first and last several rows of $\mathbf{A}$. The matrix is real, square, banded, nondefective, and non-normal. Since $\mathbf{A}$ is an autonomous operator, the solution to Eq. (12) is given by

$$
\boldsymbol{G}(t)=\exp (\mathbf{A} t) \boldsymbol{G}_{0},
$$


where $\boldsymbol{G}_{0}(\xi)$ is a vector representing the initial state of the system.

It follows that the maximum possible amplification over time $t$ is given by

$$
\beta_{\max }(t) \equiv \sup _{\boldsymbol{G}_{0} \neq 0} \frac{\|\boldsymbol{G}\|}{\left\|\boldsymbol{G}_{0}\right\|}=\|\exp (\mathbf{A} t)\|,
$$

where $\|\cdot\|$ denotes the Euclidean or two norm. Since $\mathbf{A}$ is nondefective, there exists a similarity transformation

$$
\exp (\mathbf{A} t)=\mathbf{S} \exp (\mathbf{\Lambda} t) \mathbf{S}^{-1},
$$

where $\Lambda$ is the diagonal matrix containing the eigenvalues of $\mathbf{A}$ in decreasing order, and $\mathbf{S}$ is the matrix whose columns are the normalized eigenvectors of $\mathbf{A}$ corresponding to each eigenvalue [27]. This identity can be used to establish the upper and lower bounds on $\left\|e^{\mathbf{A} t}\right\|$ according to

$$
\begin{aligned}
\exp \left(\lambda_{\max } t\right) & \leqslant\|\exp (\mathbf{A} t)\|=\left\|\mathbf{S} \exp (\mathbf{\Lambda} t) \mathbf{S}^{-1}\right\| \\
& \leqslant\|\mathbf{S}\|\left\|\mathbf{S}^{-1}\right\| \exp \left(\lambda_{\max } t\right) .
\end{aligned}
$$

For a normal operator $\mathbf{A}, \mathbf{S}$ is unitary, and both the upper and lower bounds on $\|\exp (t \mathbf{A})\|$ equal $\exp \left(\lambda_{\max } t\right) \forall t$. The eigenvalue with largest real part is, therefore, physically determinant since the growth rate of any disturbance is bounded above by $\lambda_{\max }$, the spectral abscissa of $\mathbf{A}$, which forms the leading entry in $\boldsymbol{\Lambda}$. For a non-normal operator, the eigenvectors are not orthogonal, and the product $\|\mathbf{S}\|\left\|\mathbf{S}^{-1}\right\|$ can be much larger than unity. For highly non-normal systems, several orders of transient amplification could occur and induce nonlinear effects, thereby invalidating the results of modal analysis. The transient behavior of the general solution $G(\xi)$ is therefore governed by the time dependence of $\|\exp (\mathbf{A} t)\|$. The optimal initial condition that attains the maximum amplification at time $t$ is determined as part of the analysis, which obviates the need to specify an initial form for the perturbation.

The singular value decomposition of the propagator $\exp (\mathbf{A} t)$,

$$
\exp (\mathbf{A} t)=\mathbf{U} \mathbf{\Sigma} \mathbf{V}^{\dagger}
$$

determines the evolution of an initial state $\boldsymbol{G}_{0}$ over the time interval $t$ [29]. The columns of the unitary matrix $\mathbf{V}$ represent the complete set of initial states; the columns of the unitary matrix $\mathbf{U}$ are orthonormal basis vectors that span the range space of final states. The elements $\sigma_{\mathrm{i}}$ of the diagonal matrix $\mathbf{\Sigma}$ which are ordered by growth, describe the amplification over time $t$ of each corresponding initial state. Note that the singular value decomposition must be calculated for each time $t$ at which the solutions $\mathbf{U}, \boldsymbol{\Sigma}$, and $\mathbf{V}$ are sought.

The maximum amplification over time $t$ is given by $\sigma_{\max }$, which is the leading singular value of $\exp (\mathbf{A} t)$ and by definition equal to the Euclidean norm of the matrix exponential. The optimal initial disturbance, which forms the first column of $\mathbf{V}$, is denoted by $\boldsymbol{V}_{\mathrm{opt}}$. As $t \rightarrow \infty$, the evolved state $\sigma_{\max } \mathbf{U}_{\text {opt }}$ corresponding to the optimal initial condition, evolves toward the eigenfunction $\boldsymbol{H}(\xi)$. The optimal initial disturbance $\boldsymbol{V}_{\text {opt }}(t \rightarrow \infty)$ evolves toward $\boldsymbol{H}_{\dagger}$, the eigenvector corresponding to the adjoint linearized operator $\mathbf{A}^{\dagger}$. This initial disturbance is the function that optimally excites the most unstable mode, $\boldsymbol{H}(\xi)$. The propagator $\exp (\mathbf{A} t)$ can also be used to determine the evolution of specific, suboptimal disturbances. This evolution may be useful in making comparison to experiments in which a specific disturbance can be generated in the laboratory or to other transient growth studies of suboptimal perturbations [30,31].

\section{RESULTS OF NONMODAL ANALYSIS}

All matrix norms and singular value decompositions were computed with MATLAB 5.3 subroutines. The boundary conditions in the precursor region were incorporated into the computations as follows. Recall from Sec. III that the choice $b=0$ enforces a van der Waals film, whose thickness vanishes as $\xi \rightarrow \infty$. For computational purposes, the location corresponding to a vanishingly small thickness was approximated by the position where the dimensional film height had thinned to $O(1 \mathrm{~nm})$. The use of longer domains resulted in a very slight increase in the values of $\|\exp (\mathbf{A} t)\|$ over an intermediate time interval but did not affect the magnitudes of the relevant quantities at the earliest times nor the asymptotic values as $t \rightarrow \infty$. Longer domains, however, can cause numerical inaccuracies since the condition number and the ratio of the largest to smallest element in the matrix $\mathbf{A}$ increases with the length of the precursor film. Since the precursor film is finite, however, disturbances can only extend as far as the domain boundary.

A survey of all available experimental data on thermocapillary-driven spreading [12] reveals that unstable flow and fingering is well established by dimensionless times $t<25$ (for $l$ and $U_{c}$ as defined in Sec. II) . For the computations presented below, the transient analysis was extended through $t=32$ to ensure capture of significant amplification and instability. By a time $t=30$, the numerical solutions corresponding to the evolved states of the optimal disturbances converge to the eigenfunctions obtained from the modal analysis in Sec. III B 1; the slope of the functions $\ln \mid \exp (\mathbf{A} t) \|$ for each wave number $q$ also equal the values of the growth rates $\beta(q)$ shown in Fig. 2.

\section{A. Amplification ratio}

Figure 3 depicts the temporal evolution of $\ln \|\exp (\mathbf{A} t)\|$ for selected wave numbers $q$ and two values of the parameter $V$. Figure 3(a) indicates that there is little transient growth for all wave numbers. By $t=5$, all the curves assume a linear form, whose slope asymptotes to the values of the growth rate obtained in Fig. 2. The solution corresponding to $q=0$ undergoes the largest transient growth at early times but the level of amplification is small. Figure 3(b) shows a similar trend except that the level of amplification is larger, an expected result since $V$ is smaller by an order of magnitude. For this case also, the slope of each curve asymptotes to the value of the growth rate obtained in Fig. 2. This convergence 


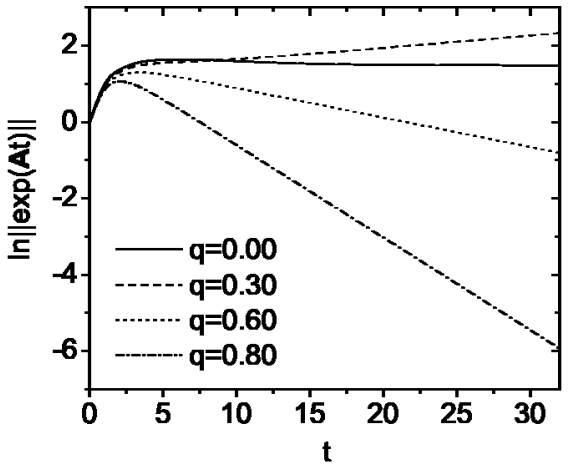

(a)

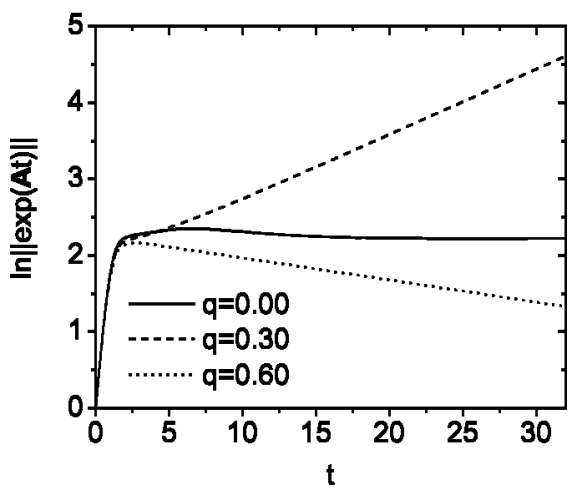

(b)

FIG. 3. Maximum possible amplification of disturbances within a time interval $t$ for (a) $V=6 \times 10^{-4}$ and (b) $V=6 \times 10^{-4}$. The ordering of disturbances by wave number $q$ according to their relative stability is in agreement with results of modal calculations. The numerical value of the slope of the curves as $t \rightarrow \infty$ reproduces the predictions of the modal analysis by a time $t=15$.

of the asymptotic nonmodal results to the predictions of the modal analysis serves as a good check on the numerical schemes used.

\section{B. Shape of optimal disturbances}

The transient analysis allows investigation of the initial and evolved form of the disturbances which induce the largest system response. This information can be used to deduce which regions of the film profile play a critical role in destabilizing the flow. Figure 4 shows the shape of the optimal initial disturbance $\boldsymbol{V}_{\text {opt }}$ and the corresponding evolved shape, $\boldsymbol{G}(t)=\sigma_{\max } \boldsymbol{U}_{\mathrm{opt}}(t)$, for $q=q_{\text {max }}=0.30, V=6 \times 10^{-4}$, and times $1 \leqslant t \leqslant 32$. The initial disturbance is applied at $t=0$. The base state solution $h_{0}(\xi)$ has been superimposed on these plots in order to visualize the regions of the film profile where the optimal disturbances get localized. The disturbance amplitudes are arbitrary since the solutions are obtained from a linear analysis but the relative ordering in magnitude signifies whether the disturbances grow or decay in time.

Examination of these curves reveals some interesting trends. The optimal initial disturbance $\boldsymbol{V}_{\text {opt }}$ applied at $t=0$ strongly localizes to the base of the capillary rim by $t=1$. This immediate and strong response lends some support to

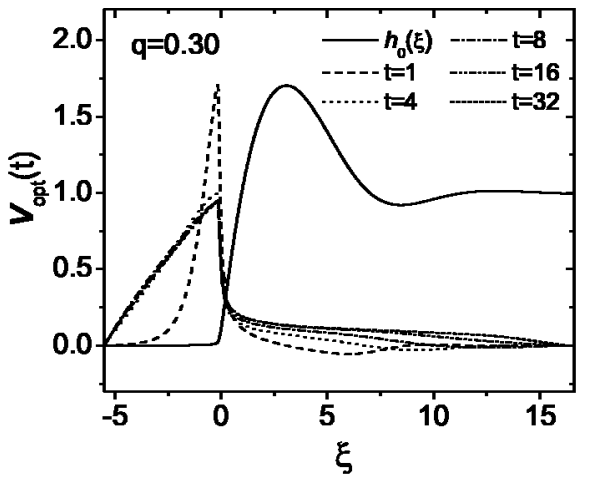

(a)

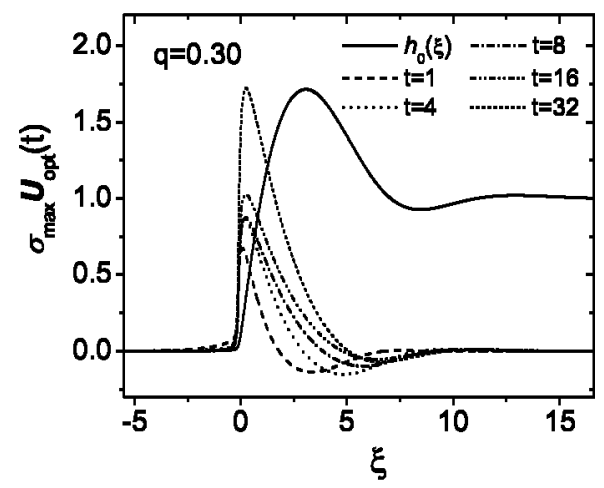

(b)

FIG. 4. Optimal initial disturbances $\boldsymbol{V}_{\mathrm{opt}}(t)$ and the corresponding evolved states $\sigma_{\text {max }} U_{\text {opt }}(t)$ for $q=0.30$ and $V=6 \times 10^{-4}$. The base state profile $h_{0}(\xi)$ is superimposed. The absolute magnitudes of the initial disturbances and evolved states are arbitrary since they are calculated from a linear theory. For convenience, these are rescaled such that the extremum of the curve with maximum amplitude equals the height of the capillary ridge in $h_{0}(\xi)$.

the interpretation of the contact line as a noise amplifier [30,31]. The optimal initial disturbances for later times, however, broaden to encompass the entire extent of the film, with the majority of the weight distributed in the precursor region. At longer times, the evolved disturbances show progressive migration into the capillary ridge. The local minimum in the oscillation also moves further back from the contact line such that the disturbance affects a larger portion of the macroscopic film. By a dimensionless time $t=32, \boldsymbol{V}_{\text {opt }}$ and $\sigma_{\text {max }} \boldsymbol{U}_{\text {opt }}$ are nearly indistinguishable from the eigenfunctions $\boldsymbol{H}_{\dagger}(\xi)$ and $\boldsymbol{H}(\xi)$, respectively. The increase in the magnitude of the evolved states over time indicates the exponential growth experienced by the initial disturbances. Plots of the optimal initial disturbances and evolved states for other choices of unstable wave number are qualitatively similar to those shown in Fig. 4. The optimal initial and final states for $V=6 \times 10^{-5}$ are also very similar to those shown in Fig. 4, the only significant difference being a slightly higher level of disturbance amplification.

Figure 5 depicts the solutions representing the initial excitation and evolved response for $q=0.60$. As shown in Fig. 2 , this wave number corresponds to an asymptotically stable mode. In contrast to Fig. 4, the solutions in Fig. 5 show that even more of the optimal initial disturbance is localized at 


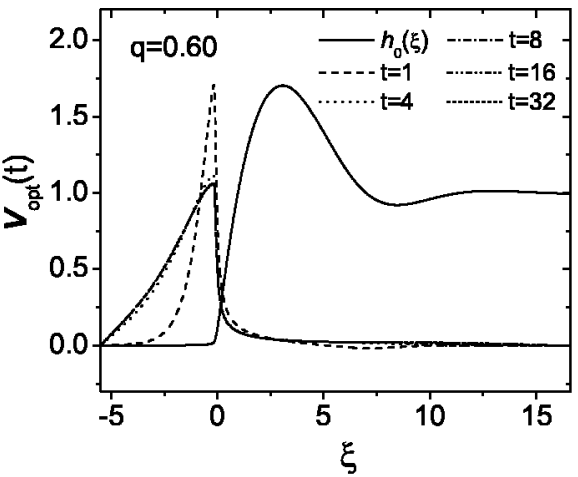

(a)

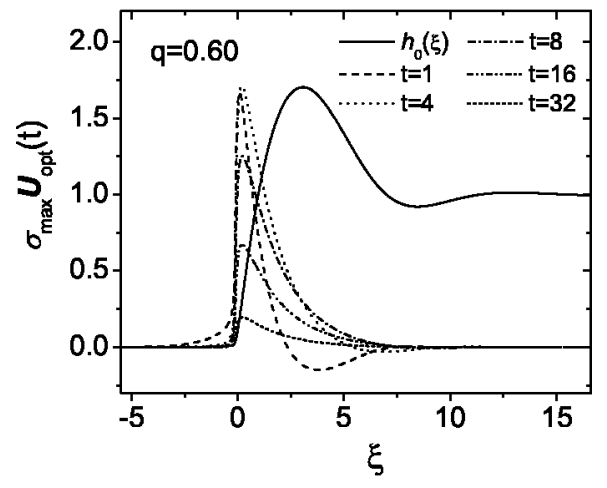

(b)

FIG. 5. Optimal initial disturbances $\boldsymbol{V}_{\mathrm{opt}}(t)$ and the corresponding evolved states $\sigma_{\max } \boldsymbol{U}_{\text {opt }}(t)$ for $q=0.60$ and $V=6 \times 10^{-4}$. The base state profile $h_{0}(\xi)$ is superimposed. The absolute magnitudes of the initial disturbances and evolved states are arbitrary since they are calculated from a linear theory. For convenience, these are rescaled such that the extremum of the curve with maximum amplitude equals the height of the capillary ridge in $h_{0}(\xi)$.

the base of the contact line and into the precursor region, with less weight apportioned to the remainder of the film behind the contact line. The exponential decay of these disturbances is also evident from the decreased magnitude of $\sigma_{\max } \boldsymbol{U}_{\text {opt }}$ at later times. Plots of the optimal initial disturbances and evolved states for other choices of stable wave number are qualitatively similar to those shown in Fig. 5.

\section{Physical interpretation of optimal initial perturbation}

The behavior of the system's response shown in Figs. 4 and 5 can be deduced by examining the position of the optimal initial disturbance relative to the position of the front of the spreading film. A disturbance well localized in the precursor region can only induce a significant response once the contact line (and eventually the capillary ridge) has made contact with the perturbation. In the moving reference frame defined by the coordinate transformation $\xi=\chi+c t$, the initial disturbance is convected toward the leading edge of the stationary film profile shown in Fig. 1(a). The disturbances that elicit the response of maximum amplitude at short times are therefore focused just ahead of the apparent contact line. At later times, these optimal disturbances broaden into the precursor region as well as the capillary ridge. The film is then continually excited during the longer residence times of the more extended disturbances. By $t \approx 15-30$ (depending on the wave number and value of $V$ ), the mode corresponding to the asymptotic modal solution assumes prominence. Since the linearized disturbance operator is non-normal, this mode is optimally excited by its associated adjoint eigenvector.

For asymptotically unstable wave numbers, the optimal disturbances that induce the largest response at short times are contained almost entirely in the precursor region, while those which induce the largest response at later times also extend through the region of the capillary ridge. The temporal behavior of these initial perturbations suggests that disturbances to the precursor region that contact the spreading film induce an immediate, amplified response, while disturbances that contact the region of the capillary ridge induce an even larger amplification which is sustained over a much longer time period. These longer lived disturbances produce the most destabilizing influence since they are present for unstable wave numbers but absent for stable ones. This hypothesis is further supported by the relative insensitivity of the results of the modal theory to the length of the precursor film used.

\section{DISCUSSION}

\section{A. Numerical issues}

While direct computation of the eigenfunctions and eigenvalues of the disturbance matrix is relatively insensitive to small inaccuracies in the base flow profile, the large number of computations needed to calculate the matrix exponential exacerbates any problems arising from the base state. The length of the precursor film can also affect the results. For a given value of $V$, shorter precursor films depress the value of the matrix norm at early times; however, the curves for $\ln \|\exp (\mathbf{A} t)\|$ vs $t$ eventually asymptote to the same slope obtained with longer precursor films for sufficiently longer times. This suppression of the amplification ratio is consistent with the interpretation of the optimal disturbances given in the preceding section. Perhaps the most serious numerical problems arise from the method by which the unperturbed film profile is matched onto the precursor film in the shooting routines. Simply patching the leading order asymptotic behavior for the precursor film shape for $b=0$ $\left(h_{0}=-6 \mathrm{~V} / \xi\right)$ onto the base flow profile yields spurious values for $\|\exp (\mathbf{A} t)\|$ that increase by several orders of magnitude per unit of dimensionless time. This inadequate matching procedure also leads to solutions for $\ln \|\exp (\mathbf{A} t)\|$, whose slope fails to asymptote to the value of $\beta(q)$ obtained from eigenanalysis. The approach followed in this work, in which the full asymptotic behavior of the precursor film [see Eq. (8)] is matched onto the base flow profile, avoids these numerical artifacts and produces the correct asymptotic behavior as $t \rightarrow \infty$.

The validity of the full matching procedure is further confirmed by comparing the results above to those obtained with a flat and infinite precursor film with van der Waals forces included (not shown). Determination of the base state profiles for these systems is less demanding numerically, so pro- 
files with sufficiently long precursor films may be obtained without using a matching scheme. The presence of a flat precursor film makes the flow more stable for a given value of $V$ and reduces the height of the capillary ridge. However, the qualitative features of the curves $\ln \|\exp (\mathbf{A} t)\|$ vs $t$ and the optimal disturbances and evolved states calculated from the singular value decomposition of $\exp (\mathbf{A} t)$ are similar to the results obtained above. Most significantly, the time dependence of $\ln \|\exp (\mathbf{A} t)\|$ is the same, and the optimal initial disturbances are similarly distributed as those shown in Figs. 4(a) and 5(a).

\section{B. Comparison of two contact line models}

Whether the precursor film used to relieve the stress singularity at the moving contact line is artificially generated through application of an infinite and uniformly thick film or more realistically generated by directly including van der Waals interactions in the governing equations, the most asymptotically unstable wave numbers and the extent of transient growth for each model are in general agreement. Accounting for the factor of $2^{1 / 3}$ difference in the scaling for $l$ [20], the van der Waals model predicts a dimensionless fingering wavelength within about one percent of the value $\lambda_{\max } / l \approx 18$ found by Kataoka and Troian [10], which favorably compares to experimentally observed values. While the specific precursor shape and thickness affect the exact level of transient disturbance amplification, the predictions for the most critical wavelength as well as the associated growth rate are insensitive to these differences. Comparison of the base state profiles, dispersion curves, and eigenfunctions generated by either the flat and infinite model or a Greenspan slip model also shows no significant difference in systems driven to spread by gravity or centrifugation provided the slip coefficient is equal to the precursor film thickness [6]. Recently, Diez, Kondic, and Bertozzi [29] concluded that both a flat precursor film and the Greenspan slip model provide an adequate numerical description of film spreading in the lubrication regime, although the use of a flat film provides some computational benefits. That work, however, did not investigate the effect of contact line models on the linear stability of the spreading film.

Both the flat precursor model [12] and the model considered here exhibit modest transient amplification of disturbances at early times even for precursor film thicknesses in the nanometer range $\left(V=6 \times 10^{-5}\right)$. The level of transient amplification differs only slightly for different wave numbers. The ordering of the transient growth curves according to the level of amplification corresponds exactly to the modal results. This smooth and rapid transition to the asymptotic behavior predicted by the modal theory explains the source of the good agreement between theory and experiment despite the non-normal property of the governing disturbance operator.

This finding of limited transient amplification differs from results for film spreading down an inclined plane [30,31]. These studies reported disturbance amplification of well over two orders of magnitude at early times $(t<10)$ regardless of the stabilizing influence of hydrostatic pressure that increases as the angle of inclination decreases. While this enhanced sensitivity to disturbances is not completely understood, there are fundamental differences between these two coating flows. The separation of scales in film thicknesses between the outer macroscopic region $\left[O\left(h_{c}\right)\right]$ and the inner precursor film region $[O(b)]$ is significantly larger in the falling film. In the thermal problem the ratio $b / h_{c}$ is $O\left(10^{-3}\right)$; in the gravity problem this ratio is typically $O\left(10^{-6}\right)$. Since the level of transient amplification increases as $b / h_{c}$ decreases, more significant transient growth is expected in the falling film. In addition, because the driving force responsible for the spreading differs significantly between the two classes of flow, the inclination angle of the solid substrate, $\theta$, has a more pronounced effect on the falling film. For the thermocapillary-driven spreading, the shear stress is a constant that is independent of $\theta$. The ratio of the hydrostatic force $\left(F_{\mathrm{h}}\right)$ to the driving force $\left(F_{\mathrm{d}}\right)$, therefore, scales as $F_{\mathrm{h}} / F_{\mathrm{d}} \sim\left(h_{c} \rho g / \tau\right) \mathrm{Ca}^{1 / 3} \cos (\theta)$ and is typically $O\left(10^{-3}\right)$. For gravitationally driven spreading, the driving force is proportional to $\sin (\theta)$, and therefore $F_{\mathrm{h}} / F_{\mathrm{d}} \sim \mathrm{Ca}^{1 / 3} \cot (\theta)$. This ratio can attain values of $O(1)$ to $O(10)$ for small inclination angles.

Modal stability predicts that the gravitationally driven flow should be stable below a critical value of $\theta$. It has been suggested [30] that perhaps this modal approach is insufficient because experiments have indicated instability even at low angles of inclination. Incorrect estimation of the film height $h_{c}$ can lead to spurious discrepancy between theory and experiment. The large amount of transient growth reported for the falling film [30] may also account for this discrepancy. The recent spectral analysis of the falling film by Ye and Chang [32] lends good insight into the mechanism responsible for the instability at small $\theta$. This analysis traces the interaction between the continuous spectrum and the discrete eigenvalues for the case of a flat and infinitely long precursor film. By contrast, no discrepancy has been observed between the predictions of the modal theory and experimental measurements for thermally driven films. In fact, the remarkably good agreement suggests that significant transient growth, which could induce nonlinear effects and thereby invalidate the asymptotic predictions of the modal theory, should not occur.

\section{CONCLUSION}

This study explores the transient amplification of optimal disturbances in thin liquid films driven to spread by thermocapillary forces. The equation governing the evolution of the film height includes an attractive van der Waals force that helps establish a precursor film, thereby removing the usual stress singularity in problems involving a moving contact line. The initial disturbances that induce the maximum possible amplification at time $t$, as well as the corresponding evolved states, are identified through a generalized stability analysis appropriate for non-normal systems. This nonnormal property of the linearized disturbance operator A requires that the upper bound on the disturbance growth rate be determined from the norm of the matrix exponential $\|\exp (\mathbf{A} t)\|$ rather than from the eigenvalue of $\mathbf{A}$ with largest 
real part, which determines the stability for all time only for normal operators.

This transient analysis yields several noteworthy results. The optimal disturbances for both asymptotically stable and unstable solutions initially localize to the contact line region near the leading edge of the capillary ridge. At later times, these perturbations broaden extensively into the precursor region. The solutions corresponding to asymptotically unstable modes, however, also migrate into the region of the capillary ridge. Computation of the maximum possible amplification, as quantified by $\ln \|\exp (\mathbf{A} t)\|$, indicates minimal transient growth with smooth and rapid transition to the growth rate $\beta(q)$ obtained from conventional modal analysis. Smaller values of the van der Waals parameter $V$ produce only slightly larger amplification ratios. This system therefore exhibits only weak non-normality, a result consistent with transient growth calculations using an alternative contact line model consisting of a flat and infinite precursor layer [12].

From a computational standpoint, the large number of floating point operations required for the computation of the matrix exponential exacerbates any small inaccuracies in the base flow profile, making the transient calculations much more sensitive to errors than the modal calculations relevant to conventional linear stability analysis. The approach followed in this work, in which the full asymptotic behavior of the precursor film [see Eq. (8)] is matched onto the base flow profile, yields the correct asymptotic behavior as $t \rightarrow \infty$. Pre- cursor films which are truncated prematurely depress the disturbance amplification for small times but have no effect on the asymptotic growth rate.

This absence of significant transient growth and the rapid approach to the asymptotic regime explain the close agreement previously obtained between theory and experiment, despite the fact that maximum growth rate was calculated from the eigenvalue of $\mathbf{A}$ with largest real part [10,11]. Significant nonmodal growth is likely absent because the angles between the dominant and subsequent eigenvectors is rather large. The lack of mode-mode coupling is likely due to the fact that the spatial variation of the base state is confined to the region of the capillary ridge near the moving contact line. As a result, the subdominant modes are unable to transfer sufficient energy to the leading eigenvector before undergoing significant attenuation by surface tension. Further studies of the dynamics of spreading caused by alternative driving forces and alternative contact line models using the more generalized stability analysis presented here will confirm whether all free surface lubrication flows display limited disturbance amplification.

\section{ACKNOWLEDGMENTS}

The authors gratefully acknowledge financial support from the Department of Defense (JMD), the National Science Foundation XYZ on a Chip program, and a Princeton University MRSEC grant.
[1] C.H. Ho and Y.C. Tai, Annu. Rev. Fluid Mech. 30, 579 (1998).

[2] M. Gad-El-Hak, J. Fluids Eng. 121, 5 (1999).

[3] D.E. Kataoka and S.M. Troian, Nature (London) 402, 794 (1999).

[4] A. A. Darhuber et al., in Micro Total Analysis Systems 2001, edited by J. M. Ramsey and A. van den Berg (Kluwer Academic, Dordrecht 2001), p. 244.

[5] S.M. Troian et al., Europhys. Lett. 10, 25 (1989).

[6] M.A. Spaid and G.M. Homsy, Phys. Fluids 8, 460 (1996).

[7] V.G. Friz and Z.F. Angew, Physik 19, 374 (1965).

[8] L.H. Tanner, J. Phys. D 12, 1473 (1979).

[9] H. Greenspan, J. Fluid Mech. 84, 125 (1978).

[10] D.E. Kataoka and S.M. Troian, J. Colloid Interface Sci. 192, 350 (1997).

[11] D.E. Kataoka and S.M. Troian, J. Colloid Interface Sci. 203, 335 (1998).

[12] D. E. Kataoka, Ph.D. thesis, Princeton University, 1999.

[13] C. Huh and L.E. Scriven, J. Colloid Interface Sci. 35, 85 (1971).

[14] A.A. Golovin, B.Y. Rubinstein, and L.M. Pismen, Langmuir 17, 3930 (2001).

[15] B.F. Farrell and P.J. Ioannou, J. Atmos. Sci. 53, 2025 (1996).

[16] A. Oron, S.H. Davis, and S.G. Bankoff, Rev. Mod. Phys. 69, 931 (1997).

[17] A.M. Cazabat, F. Heslot, S.M. Troian, and P. Carles, Nature (London) 346, 824 (1990).
[18] J.B. Brzoska, F. Brochard-Wyart, and F. Rondelez, Europhys. Lett. 19, 97 (1992).

[19] A. W. Adamson, Physical Chemistry of Surfaces, 5th ed. (Wiley, New York, 1990), p. 53.

[20] The quantity $U_{c}$ differs by a factor of 2 from the quantity defined in Refs. [10] and [11], which used the height averaged speed instead of the surface speed for characterizing the thermocapillary flow.

[21] X. Fanton, A.M. Cazabat, and D. Quéré, Langmuir 12, 5875 (1996).

[22] P. Carles and A.-M. Cazabat, J. Colloid Interface Sci. 157, 196 (1993).

[23] P. Carles, S.M. Troian, A.M. Cazabat, and F. Heslot, J. Phys.: Condens. Matter 2, SA477 (1990).

[24] V. Ludviksson and E.N. Lightfoot, AIChE J. 17, 1166 (1971).

[25] J.A. Moriarty, L. Schwartz, and E.O. Tuck, Phys. Fluids A 3, 733 (1991).

[26] R. T. Goodwin III, Ph.D. thesis, Stanford University, 1991.

[27] G. H. Golub and C. F. V. Loan, Matrix Computations, 2nd ed. (Johns Hopkins University Press, Baltimore, MD, 1990).

[28] L. N. Trefethen and D. Bau III, Numerical Linear Algebra (SIAM, Philadelphia, PA, 1997).

[29] J.A. Diez, L. Kondic, and A. Bertozzi, Phys. Rev. E 63, 011208 (2000).

[30] A.L. Bertozzi and M.P. Brenner, Phys. Fluids 9, 530 (1997).

[31] L. Kondic and A.L. Bertozzi, Phys. Fluids 11, 3560 (1999).

[32] Y. Ye and H.-C. Chang, Phys. Fluids 11, 2492 (1999). 